UDC 519.6

\title{
Linear combination of the Upwind and Standard Leapfrog difference schemes with weight coefficients obtained by minimizing the approximation error *
}

\author{
A.E. Chistyakov, A. M. Atayan \\ Don State Technical University, Rostov-on-Don, Russia
}

In order to solve the transfer problem, it is proposed to use the scheme based on a linear combination of the Upwind and Standard Leapfrog difference schemes with weight coefficients obtained by minimizing the approximation error. The estimate of the approximation error of the proposed difference scheme shows that, for small Courant numbers, this scheme whose approximation error is $O(\operatorname{ch} 2)$, where the constant c is significantly less than unity, is preferable to use than the original Upwind and Standard Leapfrog schemes whose approximation errors are $O(h 2)$. The proposed modification of the Upwind Leapfrog difference scheme is effective for solving the diffusion-convection problem in the range of Peclet numbers $2 \leq P e \leq 20$.

Keywords: Navier-Stokes equation, splitting schemes for physical processes, the Upwind and Standard Leapfrog difference schemes with weight coefficients.

Introduction. The problem of large Peclet numbers occurs $[7,13]$ when solving problems of transporting suspensions of shallow water bodies $[2,4,11]$ based on central-difference schemes. One of the solutions to this problem is grinding a step on a spatial grid, which entails an increase in labor intensity. When solving the three-dimensional diffusion-convection problem, in order to reduce the Peclet number by two times, it is necessary to reduce the steps in space by two times, and in time by four times. Thus, the complexity increases by 32 times. Another approach to solving this class of problems is the use of other difference schemes, for example, the Upwind Leapfrog scheme. The Upwind Leapfrog schemes with limiters have shown their effectiveness in solving problems of aeroacoustics [24, 25]. In [18], it was proposed to use a linear combination of the Upwind and Standard Leapfrog schemes. The optimal coefficients for this scheme were calculated in [15] from the condition of minimization of the order of approximation error [21-23]. The purpose of this work is to determine the range of effectiveness of the application of this scheme, using a linear combination of the Upwind and Standard Leapfrog schemes with weighting coefficients obtained from the condition of minimizing the order of approximation error.

\section{Accuracy of solving the heat conduction problem.}

Formulation of the problem. Formulation of the problem. Consider the case of the heat equation with constant coefficients.

$$
q_{t}^{\prime}=\mu q_{x x}^{\prime \prime}+f, \quad t>0, \quad 0<x<l,
$$

\footnotetext{
${ }^{*}$ The work was supported by the RFBR (project No. 19-07-00623).
} 


$$
\begin{aligned}
& \left.q(x, t)\right|_{t=0}=q^{0}(x), \quad 0 \leq x \leq l, \\
& q(0, t)=q_{0}(t), \quad q(l, t)=q_{l}(t), \quad t \geq 0 .
\end{aligned}
$$

Negotiation of initial and boundary conditions:

$$
q^{0}(0)=q_{0}(0), \quad q^{0}(l)=q_{l}(0) .
$$

Analytical solution of the diffusion equation. Let us find an analytical solution of the problem (1). It should be noted that in the case of a table method for determining the value $u_{0}$ the series will be limited by $N-1$ harmonics, as for the restoration of a continuous function, an interpolation trigonometric polynomial is used, where $N$ is the number of discrete values of the function.

Functions $q$ and $f$ can be represented as rows:

$$
q \square \sum_{m=1}^{N-1} C_{m}^{(q)}(t) \sin (\omega m x), f \square \sum_{m=1}^{N-1} C_{m}^{(f)} \sin (\omega m x),
$$

where $\omega=\pi / l, C_{m}^{(f)}=\frac{2}{l} \int_{0}^{l} f(x) \sin (\omega m x) d x, C_{m}^{(q)}=\frac{2}{l} \int_{0}^{l} q(x) \sin (\omega m x) d x$.

It should be noted that for the functions with period $2 \pi$, having $\alpha$-ht derivative satisfying the inequality $f^{(\alpha)}(x) \leq K$.

There is an estimate of the residual term of the series (4) for any integer value $\alpha$ : $\sup |r|=\frac{4 K}{\pi^{2}} \frac{\ln n}{n^{\alpha}}+O\left(1 / n^{\alpha}\right)$, where $r=u(x / \omega, t)-\sum_{m=1}^{N-1} C(t) \sin (m x)$.

Functions $u$ and $f$ substitute in the heat equation (1) and we get:

$$
\left(\sum_{m=1}^{N-1} C_{m}^{(q)} \sin (\omega m x)\right)_{t}^{\prime}=\mu\left(\sum_{m=1}^{N-1} C_{m}^{(q)} \sin (\omega m x)\right)_{x x}^{\prime \prime}+\sum_{n=1}^{N-1} C_{m}^{(f)} \sin (\omega m x) .
$$

Changing the operation of differentiation with the summation of a series and calculating the derivative in space, we obtain:

$$
\sum_{m=1}^{N-1}\left(C_{m}^{(q)}(t)\right)_{t}^{\prime} \sin (\omega m x)=\sum_{m=1}^{N-1} \mu C_{m}^{(q)}\left(-\omega^{2} m^{2} \sin (\omega m x)\right)+\sum_{n=1}^{N-1} C_{n}^{(f)} \sin (\omega m x) .
$$

Considering the linear independence of functions $\sin (\omega m x)$, we obtain:

$$
\left(C_{m}^{(q)}(t)\right)_{t}^{\prime}=-\mu \omega^{2} m^{2} C_{m}^{(q)}+C_{m}^{(f)} .
$$

The solution of equation (5) takes the form: 


$$
C_{m}^{(q)}(t)=\left(C_{m(0)}^{(q)}-\frac{C_{m}^{(f)}}{\mu \omega^{2} m^{2}}\right) e^{-\mu \omega^{2} m^{2} t}+\frac{C_{m}^{(f)}}{\mu \omega^{2} m^{2}}
$$

After the transformations and calculations, taking into account the given initial and boundary conditions, the desired function will be found [8]:

$$
q=\sum_{m=1}^{N-1}\left(\left(C_{m(0)}^{(q)}-\frac{C_{m}^{(f)}}{\mu \omega^{2} m^{2}}\right) e^{-\mu \omega^{2} m^{2} t}+\frac{C_{m}^{(f)}}{\mu \omega^{2} m^{2}}\right) \sin (\omega m x)
$$

Difference scheme for the heat equation. To solve problem (1) numerically, we will cover the computational domain with a uniform grid:

$$
w_{h}=\left\{t^{n}=n \tau, x_{i}=i h ; n=\overline{0 . . N_{t}}, i=\overline{0 . . N_{x}} ; N_{t} \tau=T, N_{x} h=l\right\} \text {, }
$$

where $\tau$ is the time step, $h$ is the space step, $M$ is the upper time limit, $N$ is the number of nodes in space.

$$
\frac{q_{i}^{n+1}-q_{i}^{n}}{\tau}=\mu \frac{q_{i+1}^{n+\sigma}-2 q_{i}^{n+\sigma}+q_{i-1}^{n+\sigma}}{h^{2}}+f_{i},
$$

where $q_{i}^{n+\sigma}=\sigma q_{i}^{n+1}+(1-\sigma) q_{i}^{n}, \sigma \in[0,1]$ is the weight of the scheme.

Stability of an explicit scheme. To study the stability we use the harmonics method for this we write the function $q_{i}^{n}$ in the form [5]:

$$
q_{i}^{n}=\lambda^{n} \cdot \exp (i j h a), j^{2}=-1,\left|e^{i j h \alpha}\right|=1 .
$$

Let us substitute (9) into (8) in the case of an explicit scheme:

$$
\begin{gathered}
\frac{\lambda^{n+1} e^{i j h \alpha}-\lambda^{n} e^{i j h \alpha}}{\tau}=\mu \frac{\lambda^{n} e^{i(j-1) h \alpha}-2 \lambda^{n} e^{i j h \alpha}+\lambda^{n} e^{i(j+1) h \alpha}}{h^{2}} \\
\text { or } \lambda-1=\mu \frac{\tau}{h^{2}}\left(e^{-i h a}-2+e^{i h a}\right) .
\end{gathered}
$$

Inasmuch as $e^{-i h a}+e^{i h a}=2 \cos (h \alpha)$, we get

$$
\lambda=1+\frac{2 \tau}{h^{2}} \mu(\cos (\mathrm{h} \alpha)-1) ; \lambda=1-\frac{4 \tau}{h^{2}} \mu \sin ^{2} \frac{h \alpha}{2}, \gamma=\frac{\tau}{h^{2}} \mu .
$$

Obviously, that $1-4 \gamma \sin ^{2} \frac{h \alpha}{2} \geq-1 \Rightarrow|\lambda| \leq 1$, as $\gamma=\frac{\tau \mu}{h^{2}}>0$.

$$
4 \gamma \sin ^{2} \frac{h \alpha}{2} \leq 2, \text { then } 4 \gamma \sin ^{2} \frac{h \alpha}{2} \leq 4 \gamma \leq 2 \Rightarrow \gamma \leq \frac{1}{2} .
$$

Thus, we obtain $\tau \leq \tau_{\max }=h^{2} / 2 \mu$. Despite the fact that this estimate is a strict limitation for explicit difference schemes, in practice, the time step must be taken even less. 
Model problem I. It is required to find a solution to the equation

$$
q_{t}^{\prime}=\mu q_{x x}^{\prime \prime}, \mu=1 \mathrm{~m}^{2} / s, 0 \leq t \leq T, 0 \leq x \leq l, q(t, 0)=0
$$

with initial conditions: $q^{0}(x)=\theta(20-x)-\theta(10-x)$, where $\theta(x)$ is the Heaviside function.

The parameters of the computational grid: the time step is in the range from 0.001 to $10 \mathrm{~s}$, the space step is $h=1 \mathrm{~m}$, the length of the time interval $T$ is $60 \mathrm{~s}$. Figure 1 shows the error in solving the model problem $I$ based on the scheme (8), 1 is a scheme with weights $(\sigma=0.5), 2$ is an explicit scheme. The error is calculated by the formula $\Psi=\sqrt{\sum_{i}\left(\tilde{q}_{i}-q_{i}\right)^{2} / \sum_{i} q_{i}^{2}}$, where $q_{i}$ is the exact value of the solution of the diffusion problem in a node $i, \tilde{q}_{i}$ is the numerical solution depending on the time step. The horizontal axis represents the value of the time step $\tau_{0}$ referred to the value $\tau_{\max }$ $\left(\tau_{0}=\tau / \tau_{\max }\right)$.

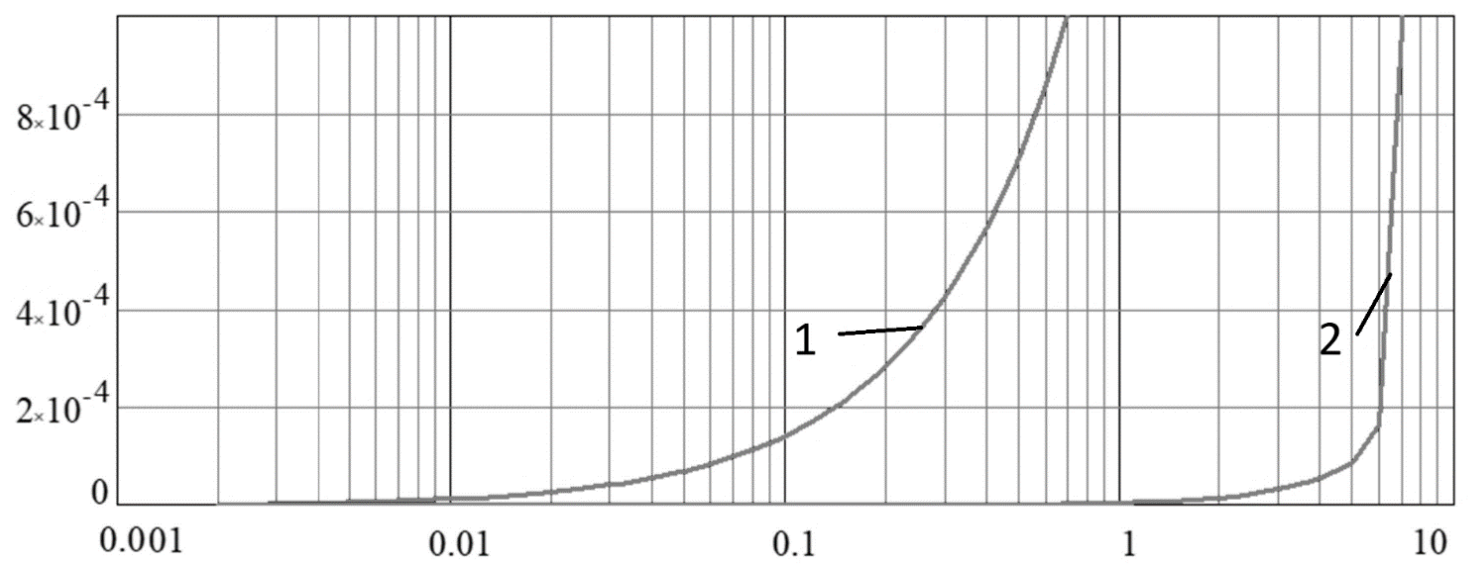

Fig. 1. The function of the dependence of the approximation error on the time step $\tau_{0}(1-$ for explicit scheme, 2 - for scheme with weights)

In order for the relative error of the explicit scheme to be $0.01 \%$, it is necessary to take the value $\tau_{0}$ equal to 0.0717 ; in the case of using the proposed scheme with weights, the parameter $\tau_{0}$ equal 5.1858.

Model problem II. Consider the problem occurs in the simulation of suspension transport in shallow water bodies $[6,9]$. It is required to find a solution to the two-dimensional diffusion equation for a region stretched in one direction.

$$
\begin{aligned}
& q_{t}^{\prime}=\mu_{x} q_{x x}^{\prime \prime}+\mu_{y} q_{y y}^{\prime \prime}, \quad t>0, \quad 0<x<l_{x}, \quad 0<y<l_{y}, \\
& \mu_{x}=100 \mathrm{~m}^{2} / s, \quad \mu_{y}=0.5 \mathrm{~m}^{2} / \mathrm{s}, \quad l_{x}=2000 \mathrm{~m}, l_{y}=5 \mathrm{~m}
\end{aligned}
$$

with initial conditions

$$
\left.q(x, y, t)\right|_{t=0}=(\theta(1100-x)-\theta(900-x))(\theta(3-y)-\theta(2-y)),
$$


$0 \leq x \leq l_{x}, 0 \leq y \leq l_{y}$ and boundary conditions in the form of Dirichlet.

The parameters of the computational grid: space steps $h_{x}=100 \mathrm{~m}$ and $h_{y}=0.5 \mathrm{~m}$, he length of the time interval $T$ is $600 \mathrm{~s}$. Fig. 2 presents the solutions of the model problem II based on: 1 - schemes with weights, 2 - explicit schemes.

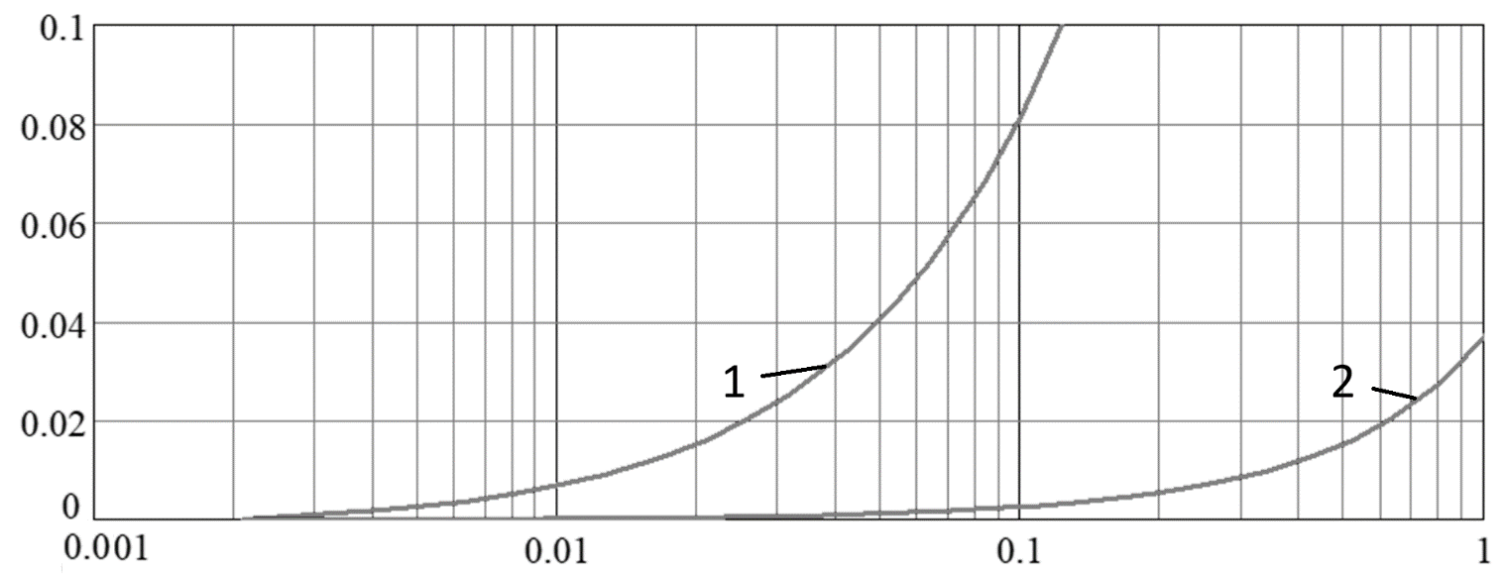

Fig. 2. The function of the dependence of the approximation error on the time step $\tau_{0}(1-$ for explicit scheme, 2 - for scheme with weights)

Figures 1, 2 shows that the error of achievement for an explicit scheme is that the limit on the time step is substantially less than for the scheme with weights. In order for the relative error of the explicit scheme to be equal to one percent, the value $\tau_{0}$ must be taken equal to 0.01376 , in the case of using the proposed scheme with weights, the parameter $\tau_{0}$ is 0.34844 .

Remark 1. An explicit scheme has a stable solution under the constraint $\tau \leq O\left(h^{2}\right)$ [26], scheme with weights with $\sigma \geq 0.5$ has no restrictions on the time. In practice, in order for the calculation error, based on a difference scheme (8) with a weight $0 \leq \sigma \leq 1$, was in an acceptable range, you must use the following limit on the time step:

$$
\tau \leq \Delta \cdot\left(\sum_{i=1}^{r} 2 \mu_{i} / h_{i}^{2}\right)^{-1},
$$

where $r$ is the dimension of space. The parameter $\Delta$ describes the ratio of the step that is necessary to take in order for the accuracy of the calculations to be in an acceptable range $\tau$ to the step obtained from the constraint on the stability of the explicit scheme $\tau_{\max }$, the assessment takes place $\tau_{0} \leq \Delta$. When solving problems of diffusion-convection, it is necessary to find values $\Delta$ differently, if the step $\tau$ is taken too large, then the error will be large, and if small, the computational effort is large. For an explicit scheme, it is recommended to take the parameter $\Delta$ equal 0.01 , and for a scheme with a weight $\sigma=0.5$, the parameter $\Delta$ can be taken equal to 0.3 .

\section{Solution of the transfer problem based on the Upwind Leapfrog scheme}

Consider the transfer equation [14] 


$$
q_{t}^{\prime}+u q_{x}^{\prime}=0
$$

where $t \in[0, T], x \in[0, l], q(0, x)=q^{0}(x), q(t, 0)=0, u=$ const .

Let introduce a uniform computational grid $\omega=\bar{\omega}_{h} \times \omega_{\tau}$, where $\bar{\omega}_{h}=\left\{x_{i} \mid x_{i}=i h, i=0,1, \ldots, N, N h=l\right\}, \omega_{\tau}=\left\{t^{n} \mid n=0,1, \ldots, T\right\}, \tau=t^{n+1}-t^{n}=$ const.

For the numerical solution of the problem, you can use the following finite-difference schemes:

- the Upwind Leapfrog scheme [16]:

$$
\begin{aligned}
& \frac{q_{i}^{n+1}-q_{i}^{n}}{2 \tau}+\frac{q_{i-1}^{n}-q_{i-1}^{n-1}}{2 \tau}+u \frac{q_{i}^{n}-q_{i-1}^{n}}{h}=0, u \geq 0 ; \\
& \frac{q_{i}^{n+1}-q_{i}^{n}}{2 \tau}+\frac{q_{i+1}^{n}-q_{i+1}^{n-1}}{2 \tau}+u \frac{q_{i+1}^{n}-q_{i}^{n}}{h}=0, u<0 ;
\end{aligned}
$$

- the Standard Leapfrog scheme:

$$
\frac{q_{i}^{n+1}-q_{i}^{n-1}}{2 \tau}+u \frac{q_{i+1}^{n}-q_{i-1}^{n}}{2 h}=0
$$

Remark 2. It is known that solving the problem (10) on the basis of central difference schemes is not stable; at the same time, for solving this class of problems, the Upwind Leapfrog scheme with limiters [17] showed its effectiveness

To solve problem (10), we will use a scheme built on the basis of a linear combination of the difference scheme Upwind and Standard Leapfrog with weighting factors of 2/3 and 1/3, respectively, obtained by minimizing the approximation error

$$
\begin{gathered}
\frac{q_{i}^{n+1}-q_{i}^{n}}{\tau}+\frac{4}{3}\left(\frac{q_{i-1}^{n}-q_{i-1}^{n-1}}{2 \tau}+u \frac{q_{i}^{n}-q_{i-1}^{n}}{h}\right)+ \\
+\frac{q_{i}^{n}-q_{i}^{n-1}}{3 \tau}+u \frac{q_{i+1}^{n}-q_{i-1}^{n}}{3 h}=0, \quad u \geq 0, \\
\frac{q_{i}^{n+1}-q_{i}^{n}}{\tau}+\frac{4}{3}\left(\frac{q_{i+1}^{n}-q_{i+1}^{n-1}}{2 \tau}+u \frac{q_{i+1}^{n}-q_{i}^{n}}{h}\right)+\frac{q_{i}^{n}-q_{i}^{n-1}}{3 \tau}+u \frac{q_{i+1}^{n}-q_{i-1}^{n}}{3 h}=0, u<0 .
\end{gathered}
$$

Model problem III. Consider the problem of movement of the front of concentrations [19, 20]. Required to find a solution to the equation

$$
q_{t}^{\prime}+u q_{x}^{\prime}=0, u=0.5 \mathrm{~m} / \mathrm{s}, 0 \leq t \leq T, 0 \leq x \leq l, q(t, 0)=0
$$


with initial conditions: $q^{0}(x)=\theta(70-x)-\theta(60-x)$.

Fig. 3. presents the values of the errors in the norm

$$
L_{1} \Psi^{n}=\sum_{i} \psi_{i}^{n} h, \psi_{i}^{n}=\left|q_{i}^{n}-q\left(x_{i}, t^{n}\right)\right|,
$$

where $q\left(x_{i}, t^{n}\right)$ is the exact solution of the problem (10) in the node $i, q_{i}^{n}$ is the numerical solution at time step n, $n=T$ ) of numerical solution of model problem III based on: the proposed scheme (13), as well as the Upwind and Standard Leapfrog schemes of with limiters depending on the values of Courant numbers $(c=|u| \tau / h)$. The length of the time interval $T$ is $100 \mathrm{~s}$. The time step $\tau$ took values of $0.02 s$ to $2 s$. Courant numbers range from 0.01 to 1 .

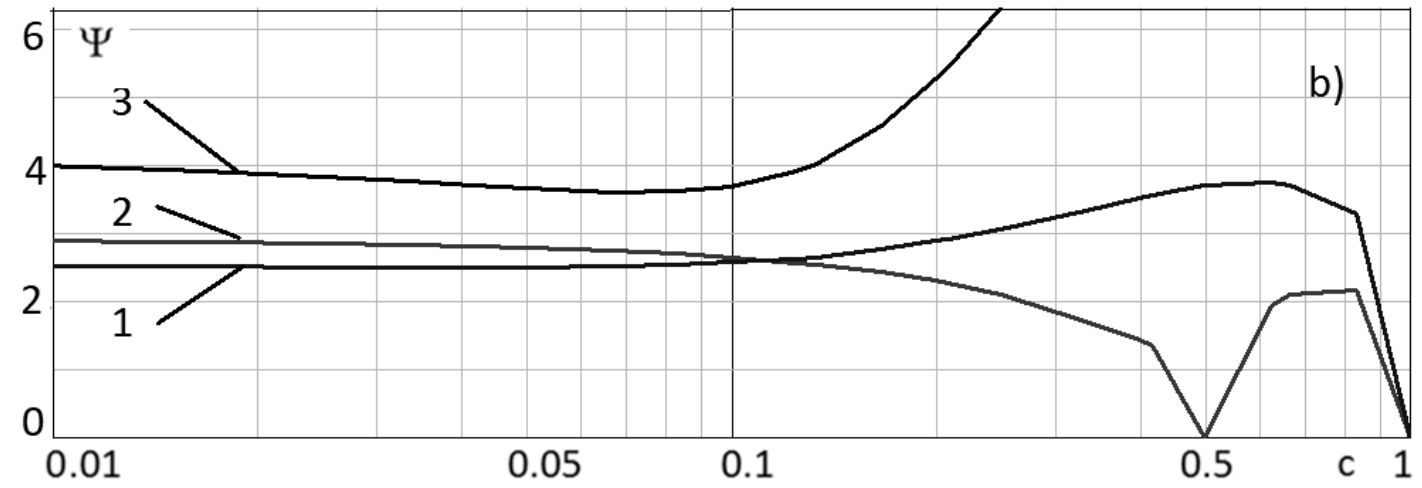

Fig. 3. The values of the errors of numerical solution of the model problem III depending on the values of Courant numbers $(1$ - schemes based on linear combination of the difference scheme Upwind and Standard Leapfrog with weight coefficients $2 / 3$ and $1 / 3$, respectively, 2 - the Upwind Leapfrog scheme with limiters and 3 - the Standard Leapfrog scheme with limiters)

Remark 3. From the results of the calculation of model problem III, it can be seen that the proposed scheme (13) more precisely the Upwind Leapfrog scheme with limiters solves the convection problem for small Courant numbers $(c=|u| \tau / h \leq 0.1)$. From the results of the calculation of the diffusion problem, it follows that for explicit schemes there is a limitation $\tau \leq \Delta \cdot \tau_{\max }, \tau_{\max }=h^{2} / 2 \mu, \Delta=0.01$. From these estimates it follows, that $\Delta|u| h / 2 \mu \leq 0.1$ or $P e=|u| h / \mu \leq 0.2 / \Delta=20$, where $P e$ is the is the grid Peclet number [13]. In this range of Peclet numbers, the proposed approximation of the convective transfer operator will be effective (the case of the absence of monotonicity of schemes constructed on the basis of central difference approximations is considered $P e>2$ ).

\section{Solution of convection-diffusion problem}

Consider the convection-diffusion equation [2,3]:

$$
q_{t}^{\prime}+u q_{x}^{\prime}=\mu q_{x x}^{\prime \prime}
$$

where $t \in[0, T], x \in[0, l]$, with boundary and initial conditions: 


$$
q(0, x)=q^{0}(x), q(t, 0)=q(t, l)=0, u=\text { const } .
$$

The approximation of the problem (14), taking into account the difference analogue of the convective transfer operator (13), is written in the form:

$$
\begin{gathered}
\frac{q_{i}^{n+1}-q_{i}^{n}}{\tau}+\frac{4}{3}\left(\frac{q_{i-1}^{n}-q_{i-1}^{n-1}}{2 \tau}+u \frac{q_{i}^{n}-q_{i-1}^{n}}{h}\right)+ \\
+\frac{q_{i}^{n}-q_{i}^{n-1}}{3 \tau}+u \frac{q_{i+1}^{n}-q_{i-1}^{n}}{3 h}=2 \mu \frac{q_{i+1}^{n}-2 q_{i}^{n}+q_{i-1}^{n}}{h^{2}}, \quad u \geq 0, \\
\frac{q_{i}^{n+1}-q_{i}^{n}}{\tau}+\frac{4}{3}\left(\frac{q_{i+1}^{n}-q_{i+1}^{n-1}}{2 \tau}+u \frac{q_{i+1}^{n}-q_{i}^{n}}{h}\right)+ \\
+\frac{q_{i}^{n}-q_{i}^{n-1}}{3 \tau}+u \frac{q_{i+1}^{n}-q_{i-1}^{n}}{3 h}=2 \mu \frac{q_{i+1}^{n}-2 q_{i}^{n}+q_{i-1}^{n}}{h^{2}}, u<0 .
\end{gathered}
$$

Model problem IV. It is required to find a solution to the equation:

$$
q_{t}^{\prime}+u q_{x}^{\prime}=\mu q_{x x}^{\prime \prime}, u=0.5 \mathrm{~m} / \mathrm{s}, \mu=\mathrm{const}, 0 \leq t \leq T, 0 \leq x \leq l
$$

with initial and boundary conditions:

$$
q^{0}(x)=\theta(70-x)-\theta(60-x), q(t, 0)=q(t, l)=0 .
$$

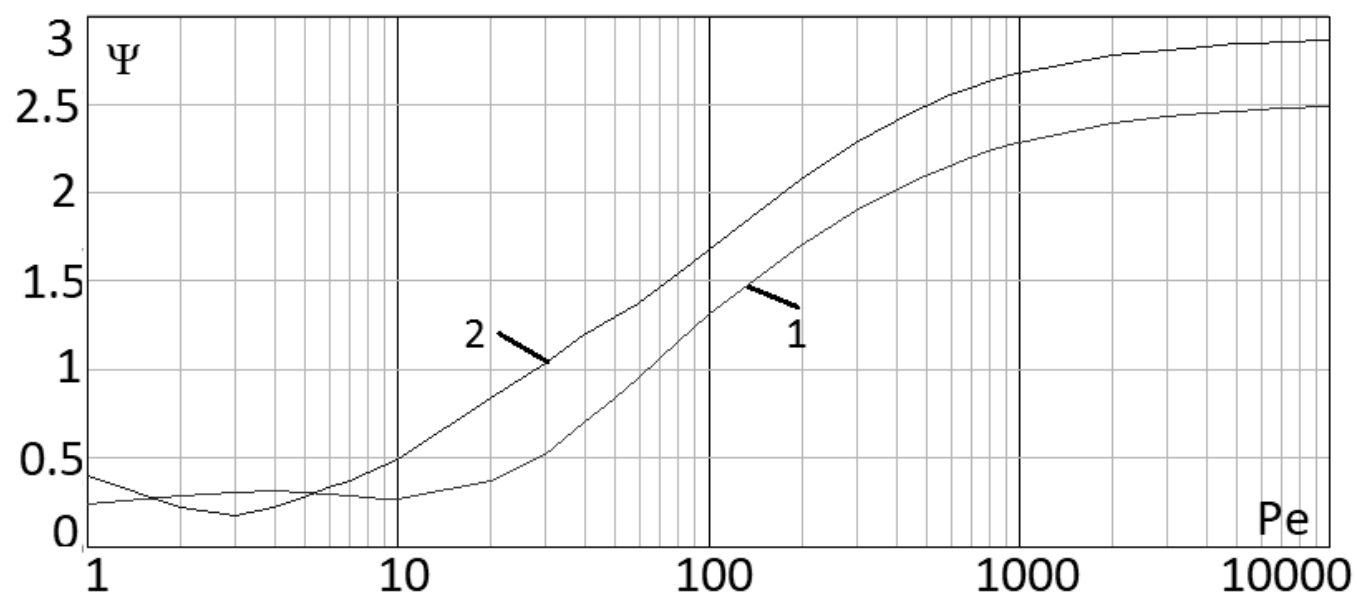

Fig. 4. The graphs of the error function $\Psi^{n}$ of the model IV solution based on the difference scheme (15) and the cabaret scheme with limiters of the solution in the $\mathrm{L}_{1}$ norm depending on the grid Peclet number

The solution of the model problem IV can be represented as [8]:

$$
q(t, x)=\sum_{m=1}^{N-1} c_{m}^{0} e^{-\mu \omega^{2} m^{2} t} \sin (\omega m x), c_{m}^{0}=\frac{2}{L} \int_{0}^{l} q^{0}(x+u t) \sin (\omega m x) d x, \omega=\frac{\pi}{l} .
$$


Fig. 4 shows the graphs of the error function $\Psi^{n}$ of solving the model problem IV based on the difference scheme (15) and the Upwind Leapfrog scheme with limiters of the solution in the $\mathrm{L}_{1}$ norm depending on the grid Peclet number. The parameters of the computational grid: space step $h=$ $1 \mathrm{~m}$, time step $\tau=0.02 \mathrm{~s}$, space interval $L=200 \mathrm{~m}$, time interval value $T$ is $100 \mathrm{~s}$, the diffusion coefficient is in the range from $5 \times 10^{-4}$ to $0.5 \mathrm{~m}^{2} / \mathrm{s}$.

Remark 4. From the results of calculating the model problem IV, it can be seen that the proposed scheme (15) has an insignificant error in the range of Peclet numbers $P e \leq 20$.

The approximation of the problem (14) on the basis of explicit central difference schemes will be written in the form $[10,12]$ :

$$
\frac{q_{i}^{n+1}-q_{i}^{n}}{\tau}+u \frac{q_{i+1}^{n}-q_{i-1}^{n}}{2 h}=\mu \frac{q_{i+1}^{n}-2 q_{i}^{n}+q_{i-1}^{n}}{h^{2}} .
$$

Fig. 5 shows the graphs of the error function $\Psi^{n}$ of solving the model problem IV based on the difference scheme (15) and the central difference scheme (16) in the $\mathrm{L}_{1}$ norm, depending on the grid Peclet number. The parameters of the computational grid: space step $h=1 \mathrm{~m}$, time step $\tau=0.02$ $s$, space interval $L=200 \mathrm{~m}$, time interval value $T$ is $100 \mathrm{~s}$, the diffusion coefficient is in the range from $5 \times 10^{-3}$ to $5 \mathrm{~m}^{2} / \mathrm{s}$.

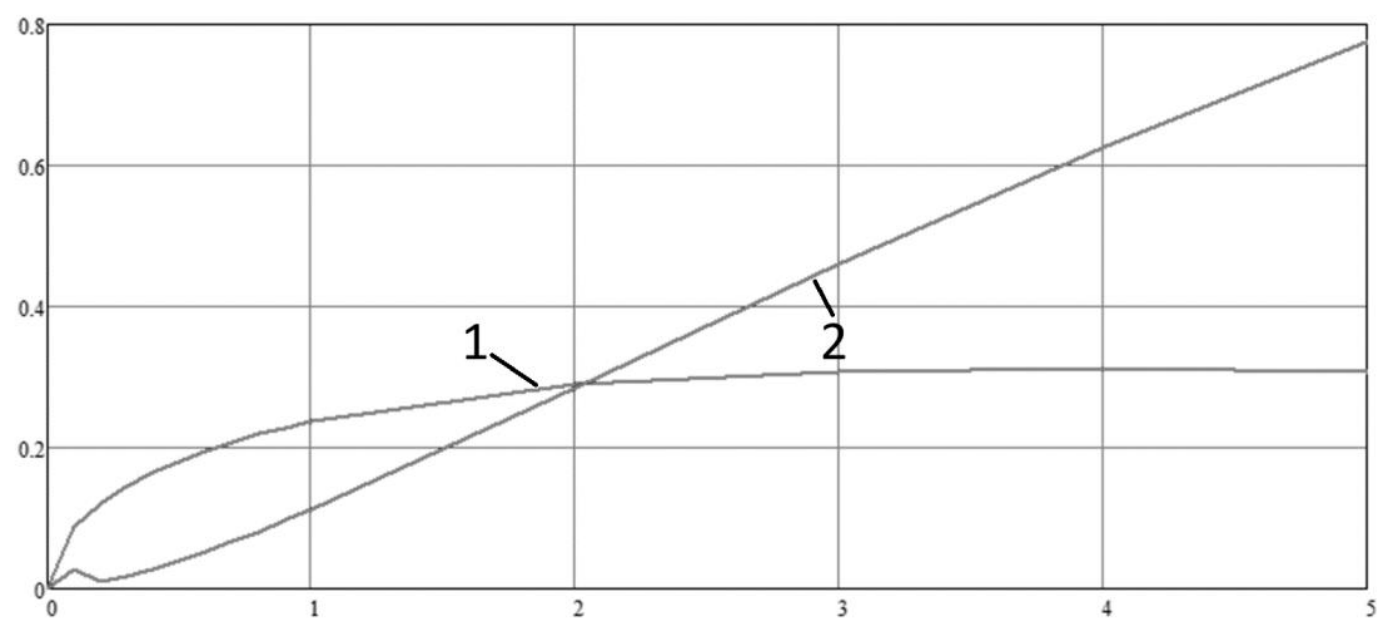

Fig. 5. The graphs of the error function $\Psi^{n}$ of solving a model problem IV based on a difference scheme (15) and a central difference scheme (16) in the $\mathrm{L}_{1}$ norm, depending on the grid Peclet number

Remark 5. From the results of the calculation of the model problem IV, it can be seen that the central difference scheme (16) has a smaller error in the range of Peclet numbers from 0 to 2 compared to the proposed difference scheme (15). Based on the above, it can be concluded that the proposed modification of the Upwind Leapfrog scheme (15) is effective for solving the diffusionconvection problem in the range of Peclet numbers $2 \leq P e \leq 20$. 
Conclusion. An explicit scheme has a stable solution under constraints $\tau \leq O\left(h^{2}\right)$, and a scheme with weights with $\sigma \geq 0.5$ has no restrictions on the time step. In practice, in order for the calculation error, based on a difference scheme with weights $0 \leq \sigma \leq 1$ to be in an acceptable range, you must use the following restriction for the time step $\tau \leq \Delta \cdot\left(\sum_{i=1}^{r} 2 \mu_{i} / h_{i}^{2}\right)^{-1}$, where $r$ is the dimension of space. The parameter $\Delta$ describes the ratio of the step that is necessary to take in order for the accuracy of the calculations to be in an acceptable range $\tau$ to the step obtained from the constraint on the stability of the explicit scheme $\tau_{\max }$, and there is an estimate $\tau_{0} \leq \Delta$. When solving problems of diffusion-convection, it is necessary to find values $\Delta$ differently, if the step $\tau$ is taken too large, then the error will be large, and if small, the computational effort is large. For an explicit scheme, it is recommended to take the parameter $\Delta$ equal 0.01 , and for a scheme with weight $\sigma=0.5$ , the parameter can be taken equal to 0.3 .

The solution of the convection problem on the basis of central difference schemes is not stable; at the same time, for solving this class of problems, the Upwind Leapfrog scheme with limiters showed its effectiveness. From the results of the calculation of model problem III, it can be seen that the proposed modification of the Upwind Leapfrog scheme is more accurate than the Upwind Leapfrog scheme with limiters for small Courant numbers $(c \leq 0.1)$. From the results of the calculation of the diffusion problem, it follows that for explicit schemes there is a limitation $\tau \leq \Delta \cdot \tau_{\max }, \tau_{\max }=h^{2} / 2 \mu, \Delta=0.01$. It follows from these estimates $P e \leq 20$, where $P e$ is the grid Peclet number.

From the results of the calculation of the model problem IV, it can be seen that the central difference scheme has a smaller error in the range of Peclet numbers from 0 to 2 compared to the proposed difference scheme. Based on the foregoing, it can be concluded that the proposed modification of the Upwind Leapfrog scheme is effective for solving the diffusion-convection problem in the range of Peclet numbers $2 \leq P e \leq 20$.

\section{References}

1. Abalakina I. V. Application of the algebraic turbulent model to the unsteady flow simulation around a cavity / I. V. Abalakina, A. N. Antonova, I. A. Graura, B. N. Chetverushkin // Matem. Mod.. - 2000. - T. 12. - № 1. - P. 45-56.

2. Alekseenko E. Coastal hydrodynamics in a windy lagoon / Alekseenko E., Roux B., Sukhinov A., Kotarba R., Fougere D. // Computers and Fluids. - 2013. - 77, P. 24-35

3. Buzalo N. Mathematical modeling of microalgae-mineralization-human structure within the environment regeneration system for the biosphere compatible city / Buzalo N., Ermachenko P., Bock T., (...), Zhmenya E., Zakharchenko N. // Procedia Engineering. - 2014. - 85, P. 84-93

4. Nikitina A.V. Optimal control of sustainable development in the biological rehabilitation of the Azov Sea / Nikitina A.V., Sukhinov A.I., Ugolnitsky G.A., (...), Puchkin M.V., Semenov I.S. // Mathematical Models and Computer Simulations. - 2017. - 9(1), P. 101-107.

5. Samarskii A. A., Numerical methods for solving convection-diffusion problems / A. A. Samarskiy, P. N. Vabischevich. - M.: Ed. URSS, 1998. - 248 P. 64 
6. Sukhinov A. Modelling of oil spill spread / Sukhinov A., Chistyakov A., Nikitina A., (...), Korovin I., Schaefer G. // 5th International Conference on Informatics, Electronics and Vision, ICIEV 2016. - 2016. - 7760176, P. 1134-1139

7. Sukhinov A.I. A mathematical model of pollutant propagation in near-ground atmospheric layer of a coastal region and its software implementation / Sukhinov A.I., Khachunts D.S., Chistyakov A.E. // Computational Mathematics and Mathematical Physics. - 2015. - 55(7), P. 1216-1231

8. Sukhinov A.I. Accuracy of the numerical solution of the equations of diffusion-convection using the difference schemes of second and fourth order approximation error / A. I. Sukhinov, A. E. Chistakov, M. V. Iakobovskii // Vestn. YuUrGU. Ser. Vych. Matem. Inform. - 2016. - Vol.5. - № 1. - P. 47-62

9. Sukhinov A.I. Complex of models, explicit regularized schemes of high-order of accuracy and applications for predictive modeling of after-math of emergency oil spill / Sukhinov A.I., Nikitina A.V., Semenyakina A.A., Chistyakov A.E. // CEUR Workshop Proceedings. - 2016. - 1576, P. 308319

10. Sukhinov A.I. Mathematical model for calculating coastal wave processes / A.I. Sukhinov, A.E. Chistyakov, E.F. Timofeeva, A.V. Shishenya // Mathematical Models and Computer Simulations. - 2013. - Vol. 5. - № 2. - P. 122-129.

11. Sukhinov A.I. Numerical realization of the three-dimensional model of hydrodynamics for shallow water basins on a high-performance system / Sukhinov A.I., Chistyakov A.E., Alekseenko E.V. // Mathematical Models and Computer Simulations. - 2011. - 3(5), P. 562-574

12. Sukhinov A.I. Reconstruction of 2001 Ecological Disaster in the Azov Sea on the Basis of Precise Hydrophysics Models (Book Chapter) // Sukhinov A.I., Sukhinov A.A. // Parallel Computational Fluid Dynamics 2004: Multidisciplinary Applications. - 2005. - P. 231-238

13. Sukhinov A.I. Solution of the matter transport problem at high Peclet number / A. I. Sukhinov, Yu. V. Belova, A. E. Chistyakov // Vychisl. Metody Programm. - 2017. - т. 18, № 4. P. 371-380.

14. Sukhinov A.I. Solution of the problem of biological rehabilitation of shallow waters on multiprocessor computer system / Sukhinov A.I., Chistyakov A.E., Levin I.I., (...), Nikitina A.V., Semenyakina A.A. //5th International Conference on Informatics, Electronics and Vision, ICIEV 2016. - 2016. - 7760175, P. 1128-1133.

15. Sukhinov A. I. CABARET difference scheme with improved dispersion properties / A. I. Sukhinov, A. E. Chistyakov // Matem. Mod. - 2019. - 31:3. - P.83-96.

16. Goloviznin V. M. Finite difference approximation of convective transport equation with space splitting time derivative / V. M. Goloviznin, A. A. Samarskii // Matem. Mod., 10:1 (1998), 86-100.

17. Goloviznin V. M. Some characteristics of finite difference scheme "cabaret" / V. M. Goloviznin, A. A. Samarskii // Matem. Mod., 10:1 (1998), 101-116.

18. Glotov V. Yu. New two-level leapfrog scheme for modeling the stochastic LandauLifshitz equations / V. Yu. Glotov, V. M. Goloviznin, S. A. Karabasov, A. P. Markeshteijn // Matem Comput. Math. Math. Phys., 54:2 (2014), 315-334.

19. Gushchin V.A. Family of quasi-monotonic finite-difference schemes of the second-order of approximation // Math. Models and Comp. Simulations, 2016, v. 8, № 5, p. 487--496. 
20. M. E. Ladonkina, O. A. Neklyudova, V. F. Tishkin Application of the RKDG method for gas dynamics problems // Math. Models and Comp. Simulations, 2014, v. 6, № 4, p. 397--407.

21. A.A. Samarskii On the regularization of difference schemes // U.S.S.R. Comput. Math. Math. Phys., 1967, v. 7, № 1, p. 62--93.

22. A.A. Samarskii Classes of stable schemes // U.S.S.R. Comput. Math. Math. Phys., 1967, v. 7, № 5, p. 171--223.

23. R.P. Fedorenko The application of difference schemes of high accuracy to the numerical solution of hyperbolic equations // U.S.S.R. Comput. Math. Math. Phys., 1963, v. 2, № 6, p. 1355-1365 .

24. Thomas, J.P. and Roe P.L., "Development of Non-Dissipative Numerical Schemes for Computational Aeroacoustics," AIAA paper 93-3382-CP, presented at the 11th AIAA Computational Fluid Dynamics Conference, Orlando, Florida, July 6-- 9, 1993.

25. S. C. Chang, X. Y. Wang, and C. Y. Chow, New Developments in the Method of SpaceTime Conservation Element and Solution Element-Applications to Two-Dimensional TimeMarching Problems, NASA TM 106758 (NASA, December 1994)

26. Boris N. Chetverushkin, "Resolution limits of continuous media models and their mathematical formulations”, Math. Models Comput. Simul., 5:3 (2013), 266-279

\section{Authors:}

Chistyakov Alexander Evgenievich, Don State Technical University (1st Gagarin Square, Rostovon-Don, Russian Federation), Doctor of Science in Physics and Maths, Associate professor

Atayan Asya Mikhailovna, Don State Technical University (1st Gagarin Square, Rostov-on-Don, Russian Federation), postgraduate student 
УДК 519.6

$10.23947 / 2587-8999-2019-1-1-1-16$

\section{Повышение гладкости численного решения моделирования задач гидродинамики на прямоугольных сетках *}

\section{А.Е. Чистяков, А.М. Атаян}

Донской государственный технический университет, Ростов-на-Дону, Российская Федерация

Для решения задачи переноса предлагается использовать схему, основанную на линейной комбинации схемы «Кабаре» и «Крест» с весовыми коэффициентами, полученными из условия минимизации порядка погрешности аппроксимации. Оценка погрешности аппроксимации предложенной разностной схемы показывает, что для малых чисел Куранта предпочтительнее использовать эту схему, погрешность аппроксимации которой равна $O(\operatorname{ch} 2)$, где константа с значительно меньше единицы, чем исходные схемы «Кабаре» и «Крест», погрешности аппроксимации которых равны $O(h 2)$. Предложенная модификация разностной схемы «Крест» эффективна для решения задачи диффузии-конвекции в диапазоне чисел пекле $2 \leq P e \leq 20$.

Ключевые слова: схемы расщепления по физическим процессам, линейная комбинация схемы «Кабаре» и «Крест» с весовыми коэффициентами.

\section{Авторы:}

Чистяков Александр Евгеньевич, Донской государственный технический университет (344000 Ростов-на-Дону, пл. Гагарина, д. 1), доктор физико-математических наук, доцент

Атаян Ася Михайловна, Донской государственный технический университет (344000 Ростов-на-Дону, пл. Гагарина, д. 1), аспирант

\footnotetext{
* Работа выполнена при поддержке РФФИ (проект № 19-07-00623).
} 